\title{
Regeneration of simple and complicated curves using Fourier series
}

\author{
Dr. Firas. Husham Al-Mukhtar \\ Computer Science Department, Knowledge University, Kurdistan Region, Iraq
}

\begin{abstract}
This paper intended to demonstrate how to regenerate the simple forms and the complicated patterns composed of sine curves using Fourier series. The result shows that the Fourier series is a preferred method over other methods described in this paper. The experimental result of the regenerated patterns proves the efficiency and the reliability of the proposed method.
\end{abstract}

Keywords-Sinusoidal Curves, least Square Regression and Fourier series.

\section{INTRODUCTION}

Many high quality and high trusted printed documents have a set of forms \& models constructed upon a family of curves and specially generated using limited functions like sinusoidal formulas. Some of these curves are boarders, guilloches and a portrait drawing found within the banknote document and stamp document.

Depending on the characteristics of the process being studied, a method of analysis could be used to extract the useful data picked with their main features to find out the hidden relationships and implied in the tabulated original form.

Different mathematical methods may be used for estimate and extract the suitable featured data of these models.

Fourier series method can be used among others like least square or latent Root Regression methods to do the extraction and the estimation of the suitable featured data needed for the reconstruction \& the regeneration of the original printed models. The Fourier series and Fourier transform (FT) is without doubt an important tool not only in the field of modern signal - and information processing, but also in other engineering sciences. For instance, Fourier transform contribute greatly to the sampling theory [1], time-frequency analysis [2], and wavelet theory [3], which constitutes the foundations of today's digital world $[4,5,6]$. One of the important results concerning Fourier series and FT is the so called Riemann- Lebesgue's Lem (RLL). Its version for the FT asserts that the FT of an integrable signal $f \in L^{1}(R)$ on the real line has a regular behavior, in the sense that it is continuous, and vanishes at infinity, i.e. it converges to 0 as $|\omega| \rightarrow \infty$ Analogously, for the Fourier series, it says that the Fourier coefficients of a w.l.o.g. $2 \pi$-periodic functions, which are each integrable on w.l.o.g $[-\pi, \pi]$, decay with increasing index toward 0 .

\section{STATISTICAL METHODS}

The model of multiple linear regressions explains the dependent variable $y_{i}$ by a linear function of $k$ explanatory variables. So in a sample of $\mathrm{n}$ observations the model can be written as [7]

$$
\begin{gathered}
\mathrm{y}=\mathrm{x} \beta+\varepsilon \quad \varepsilon)=\mathrm{O} \\
\mathrm{E}\left(\varepsilon \varepsilon^{\prime}\right)=\sigma^{2} \text { In }
\end{gathered}
$$

Where $\mathrm{x}$ matrix of explanatory variables with $\mathrm{n} \times \mathrm{k}, \mathrm{y}$ vector of dependent variable with $n \times 1, \beta$ vector of parameter with $\mathrm{k} \times 1$ and $\quad \varepsilon$ is a vector of error with $\mathrm{n} \times$ 1.

The least squares estimation (the points estimate) of $\beta$ by minimizes [7]

$$
\mathrm{s}=(\mathrm{y}-\mathrm{x} b)^{\prime}(\mathrm{y}-\mathrm{x} b)
$$

So $\hat{\beta}$ can be estimate by

$$
\hat{\beta}=\left(x^{\prime} x\right)^{-1} x^{\prime} y
$$

The matrix of $\mathrm{x}$ must have rank $\mathrm{k}$. The unbiased estimator for $\sigma^{2}$ is

$\mathrm{s}^{2}=\frac{\mathrm{e}^{\prime} \mathrm{e}}{(\mathrm{n}-\mathrm{k})}$

Where $e$ is a vector or error with $n \times 1 \quad e=(y-x \hat{\beta})$ to measure the degree of linear association between a vector $\mathrm{y}$ and a set of $\mathrm{k}$ vectors $\mathrm{x}$ we use the coefficient of determination which define by $\mathrm{R}^{2}$ with equation [7]

$$
\mathrm{R}^{2}=1-\sum_{\mathrm{i}=1}^{\mathrm{n}} \mathrm{e}_{\mathrm{i}}^{2} / \sum_{i=1}^{n}\left(y_{i}-\overline{\mathrm{y}}\right)^{2}
$$

So, some types forms for simple and multiple linear Regression can define like [7]

$$
\begin{aligned}
& 1- \\
& \mathrm{y}_{\mathrm{i}}
\end{aligned}=\beta_{0}+\beta_{1} \mathrm{x}_{1}+\beta_{2} \mathrm{x}_{2}^{2}+\ldots+\beta_{\mathrm{k}} \mathrm{x}_{\mathrm{k}}^{\mathrm{k}}+\varepsilon_{\mathrm{i}}
$$




$$
\begin{aligned}
2-y_{i} & =\beta_{0} x_{1}^{\beta_{1}} x_{2}^{\beta_{2}} \varepsilon_{i} \\
3-y_{i} & =\beta_{0}+\beta_{1} x_{1}+\beta_{2} x_{2}+\ldots+\beta_{k} x_{k}+\varepsilon_{i} \\
4-y_{i} & =\frac{1}{\alpha}+\beta_{i} x_{i}+\varepsilon_{i}
\end{aligned}
$$

Some basic assumptions for model (1) are not satisfied so we can't use equation (3) to estimate the vector $\beta$ [7].

If the model define with

$$
\begin{gathered}
\mathrm{y}=\mathrm{x} \beta+\varepsilon \quad \mathrm{E}(\varepsilon(=\mathrm{O} \\
\mathrm{E}\left(\varepsilon \varepsilon^{\prime}\right)=\mathrm{W} \sigma^{2}
\end{gathered}
$$

(6)

Where $\mathrm{W}$ is a sale matrix and $\mathrm{w}^{-1}$ exist the estimates for vector $\beta$ in model (6) when apply the method of least squares. Define by [8]

$$
\widetilde{\beta}=\left(x^{\prime} w^{-1} x\right)^{-1} x^{\prime} w^{-1} y
$$

This is a generalized least squares (GLS) estimator of vector $\beta[8]$.

In nonlinear regression model which is nonlinear in the unknown coefficient vector $\beta$ there are many types of nonlinear model like [8]

1- The univariate nonlinear regression model with function

$$
\mathrm{y}_{\mathrm{i}}=\mathrm{h}\left(\mathrm{x}_{\mathrm{i}}, \theta\right)+\varepsilon_{\mathrm{i}} \quad \mathrm{i}=1,
$$

Where $\mathrm{h}$ is a nonlinear function.

2- The M-Variate nonlinear regression model with function

$$
\begin{array}{r}
y_{i j}=h_{j}\left(x_{i}, \theta\right)+\varepsilon_{i j} \quad \begin{array}{l}
i \\
\text { ij }
\end{array} \quad 1,2, \ldots \ldots \ldots, n \\
j=1,2, \ldots \ldots \ldots, m
\end{array}
$$

So, there is $\mathrm{M}$ of these regression equations explaining $\mathrm{n}$ dependent Variable.

3- A system of nonlinear simultaneous equations.

The model define by

$$
\Phi_{\mathrm{j}}\left(\mathrm{y}_{\mathrm{i}}, \mathrm{x}_{\mathrm{j}}, \theta_{\mathrm{i}}\right)=\varepsilon_{\mathrm{ij}} \quad \mathrm{j}=1,2, \ldots \ldots, \mathrm{m}
$$

In this model the equation $j$ the $i$ th endogenous Variable is an explicit function of other endogenous Variable [9].

For model (8) the function $\mathrm{L}_{\mathrm{n}}$ to be minimized define as

$\mathrm{L}(\theta)=\frac{1}{2 \mathrm{n}} \sum_{\mathrm{i}=1}^{\mathrm{n}}\left(\mathrm{y}_{\mathrm{i}}-\mathrm{h}\left(\mathrm{x}_{\mathrm{i}}, \theta\right)\right)^{2}$

For simply we define the model (4) like $\mathrm{y}=\mathrm{h}(\theta)+\varepsilon$

Where $\mathrm{y}$ is $\mathrm{n} \times 1$ Vector of depending Variable, $\mathrm{h}(\theta)$ is a $\mathrm{n} \times 1$ Vector consisting a nonlinear function with independent Variable $\quad \mathrm{X}_{\mathrm{i}} \quad$ like $\mathrm{h}\left(\mathrm{x}_{1}, \theta\right), \mathrm{h}\left(\mathrm{x}_{2}, \theta\right), \ldots . ., \mathrm{h}\left(\mathrm{x}_{\mathrm{n}}, \theta\right)$, by linearizing the model in (12)

$$
\mathrm{y}=\mathrm{h}\left(\theta_{0}\right)+\frac{\partial \mathrm{h}\left(\theta_{0}\right)}{\partial \theta^{\prime}}\left(\theta-\theta_{0}\right)+\varepsilon
$$

Then the approximate relations to estimate the parameter depending on (GLS) method like

$\hat{\theta} \simeq \theta_{0}+\left(z^{\prime} z\right)^{-1} z^{\prime} \varepsilon$

Where

$\mathrm{z}=\frac{\partial \mathrm{h}\left(\theta_{0}\right)}{\partial \theta^{\prime}}$

To maximize a function $\mathrm{L}(\theta)$ which has a continuous first and second derivatives with respect to the Vector $\theta$, there is many Numerical methods of maximization like [10].

1- The gradient algorithm with equation

$\theta_{1}=\theta_{0}+\mathrm{k}_{0} \quad \mathrm{H}_{0} \mathrm{f}\left(\theta_{0}\right)$

Where

$$
\begin{aligned}
& f\left(\theta_{0}\right) \text { the gradient evaluated at } \theta_{0} \\
& k_{0} \text { a scalar } \\
& H_{0} \text { matrix of information }
\end{aligned}
$$

2- The method of steepest ascent

$$
\text { for equation (15) } \mathrm{H}_{0}=\mathrm{I} \text { (unit matrix) }
$$

$\mathrm{k}_{0}$ may be expensive to compute since it requires the matrix $\frac{\partial \mathrm{f}\left(\theta_{0}\right)}{\partial \theta^{\prime}}$ of the second partials of $\mathrm{L}$ with respect to $\theta$

3- The Newton-Raphson method with $\mathrm{H}_{0}=\left[\frac{\partial \mathrm{f}\left(\theta_{0}\right)}{\partial \theta^{\prime}}\right]^{-1}$ and when $\mathrm{k}=1$ the equation which gives the maximum of $\mathrm{L}(\theta)$ because.

$$
\theta_{1}=\theta_{0}-\left[\frac{\partial \mathrm{f}\left(\theta_{0}\right)}{\partial \theta^{\prime}}\right]^{-1} \mathrm{f}\left(\theta_{0}\right)
$$

4- The Gauss-Newton method is an approximation to the Newton-Raphson method in that when the matrix of second derivatives of $\mathrm{L}$ is computed. 
5- The method of scoring the Newton-Raphson method for maximizing the average log likelihood $\frac{1}{\mathrm{n}} \sum_{\mathrm{t}=1}^{\mathrm{n}} \log \mathrm{L}\left(\mathrm{y}_{\mathrm{t}}, \theta\right)$ uses as $\mathrm{H}_{0}$

6- The method of quadratic hill-climbing, in equation (15) $-\mathrm{H}_{0}$ can be replace with

$$
\left[\frac{\partial \mathrm{f}\left(\theta_{0}\right)}{\partial \theta^{\prime}}-\alpha \mathrm{I}\right]^{-1}
$$

Where $\alpha$ is a scalar is chosen to maximize $\mathrm{L}(\theta)$, the method requires computing the characteristic roots of the matrix $\frac{\partial \mathrm{f}\left(\theta_{0}\right)}{\partial \theta^{\prime}}$

\section{APPLICATION PART}

Fourier method is used to determine the coefficients of a Fourier series which approximate periodic time series data which can be defined as [11]:

$$
\begin{gathered}
i=1,2, \ldots \ldots \ldots, n \\
\quad X_{i j} \\
j=1,2, \ldots \ldots ., r
\end{gathered}
$$

Where $r$ is the number of Replication and $n$ is the number of observation for any Replicate so $X_{i j}$ is
Replicated values for $\mathrm{i}^{\text {th }}$ observation in $\mathrm{j}^{\text {th }}$ Replication the formula for Fourier harmonic model can be define [11]

$$
\hat{\mathrm{X}}_{\mathrm{i}}=\mathrm{A}_{0}+\sum_{\mathrm{k}=1}^{\mathrm{m}}\left(\mathrm{A}_{\mathrm{k}} \cos \frac{2 \Pi \mathrm{K}(\mathrm{i}-1)}{\mathrm{n}}+\beta_{\mathrm{k}} \sin \frac{2 \Pi \mathrm{K}(\mathrm{i}-1)}{\mathrm{n}}\right)
$$

The formula for determining estimates can be rewritten as follows using Semi-amplitude and phase angle like [12-14].

$$
\hat{\mathrm{X}}_{\mathrm{i}}=\mathrm{A}_{0}+\sum_{k=1}^{m}\left\{\mathrm{~V}_{\mathrm{k}} \sin \left(\frac{2 \Pi \mathrm{K}(\mathrm{i}-1)}{\mathrm{n}}+\phi_{\mathrm{k}}\right)\right\}
$$

Where Semi-amplitude and phase angle like [12-14]

$$
\begin{aligned}
& V_{k}=\sqrt{\mathrm{A}_{\mathrm{k}}^{2}+\mathrm{B}_{\mathrm{k}}^{2}} \\
& \phi_{k}=\tan ^{-1}\left(\mathrm{~A}_{\mathrm{k}} / \mathrm{B}_{\mathrm{k}}\right) \quad \mathrm{k}=1,2,3, \ldots, \mathrm{m}
\end{aligned}
$$

Tue following figures give as example of sine function which is a part of Fourier model the main aim of the research was to build w sine ware model (equation) using the method is part (1). Then use the build model to graph any sine graph [12-14].

Fig.1: example for sine wave function

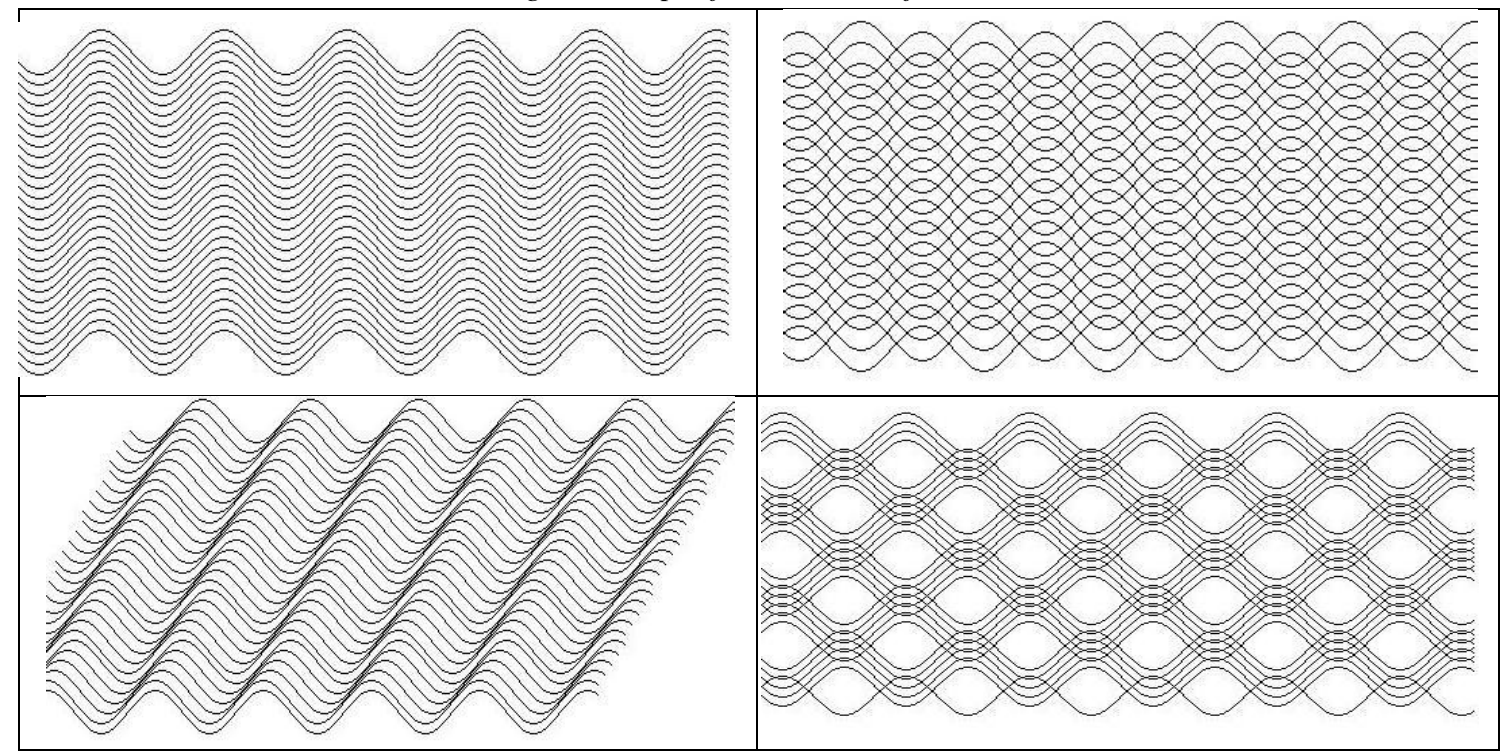



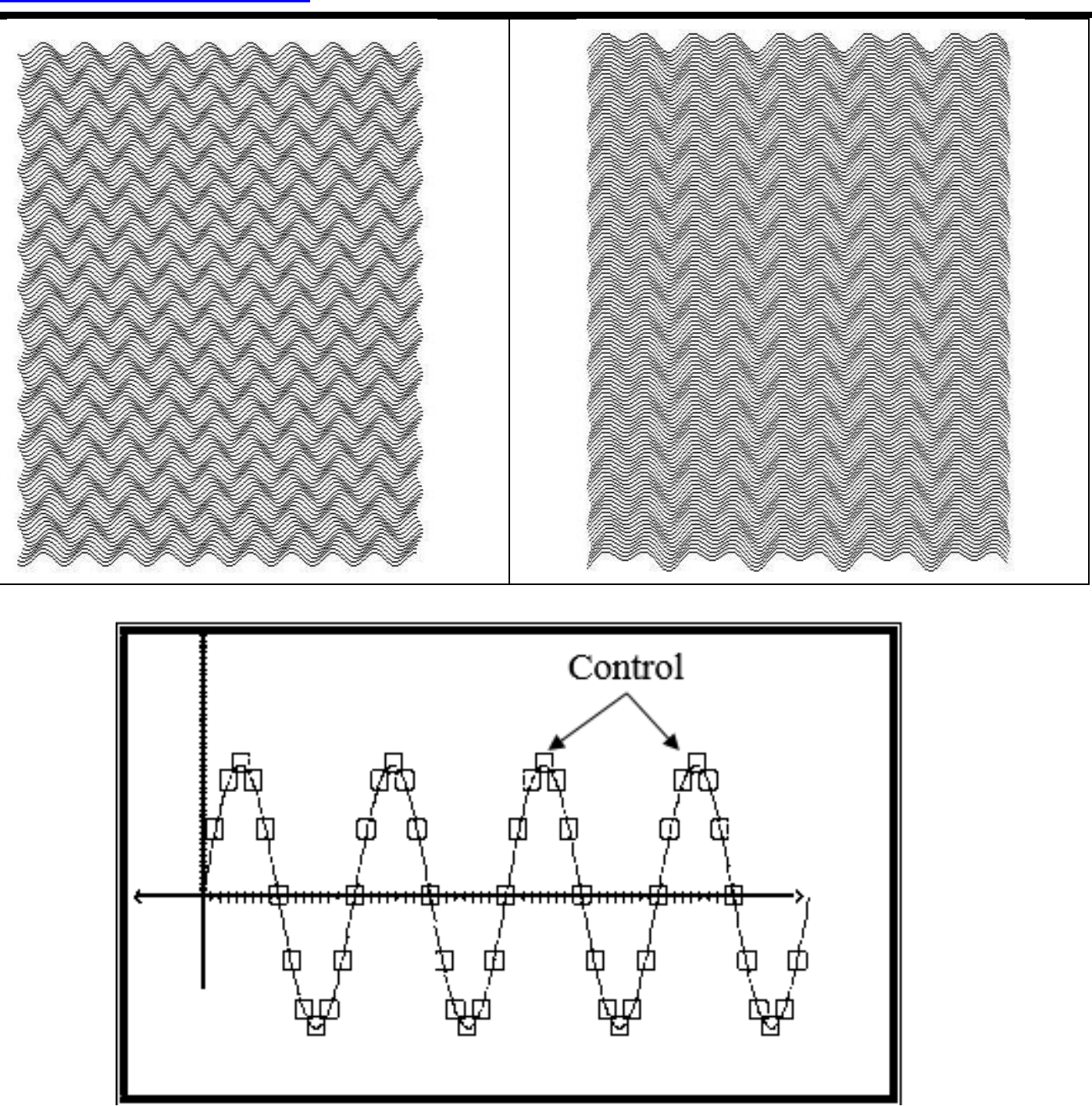

Let us have the following control point is periodic in lag equal to 22 and we want to estimate the Fourier model with min error [12-14].

Table.1: The sine wave control point

\begin{tabular}{|c|c|c|c|c|c|c|c|c|c|c|c|c|}
\hline 1.1 & 1.9 & 2.7 & 3.2 & 4.0 & 4.2 & 5.0 & 5.6 & 5.9 & 6.3 & 6.3 & 6.3 & \\
\hline 6.2 & 6.0 & 5.8 & 5.4 & 5.0 & 4.4 & 4.2 & 3.9 & 3.2 & 2.7 & 2.2 & 1.6 & 0.82 \\
\hline 0.25 & -0.4 & -1.01 & -1.5 & -1.9 & -2.25 & -2.6 & -2.6 & -2.7 & -2.5 & -1.9 & -1.65 & \\
\hline
\end{tabular}

$-1.20 \quad-0.75$

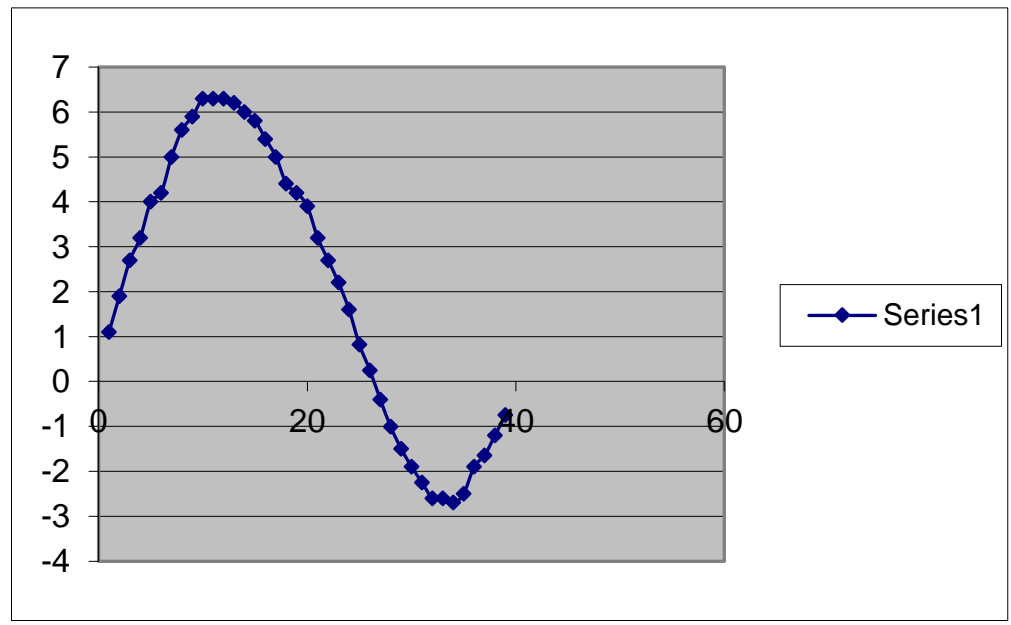


Then by using Mini-Tab package we write a program to estimate the model (the Fourier harmonic model) with harmonic equal to 4 and by using the method in part 1 we estimate the model with following parameter [12-14].

$\begin{array}{ccc}\text { K } & \text { Semi-Amplitude } & \text { Phase angle } \\ 1 & 4.38 & -0.34 \\ 2 & 0.46 & 0.11 \\ 3 & 0.12 & 0.85 \\ 4 & 0.18 & 0.23\end{array}$

Where $\quad A_{0}=\sum_{i=1}^{m} \sum_{j=1}^{n} x_{i j} / n x r$

Then we estimate the harmonic function from the observation give in table (1) and plot it with actual control point data like in figure (2) the result show the model was good representing for the data and can be use to make a sine wave plot with small error [12-14].

Table.2: The estimate data from sine model

\begin{tabular}{ccccccccccccc}
\hline 0.75 & 1.77 & 2.68 & 3.41 & 3.95 & 4.35 & 4.72 & 5.11 & 5.52 & 5.96 & 6.30 & 6.41 \\
\hline 6.34 & 6.14 & 5.80 & 5.43 & 5.02 & 4.70 & 4.31 & 3.82 & 3.28 & 2.75 & 2.15 & 1.61 \\
\hline 0.88 & 0.25 & -0.40 & -1.1 & -1.61 & -1.92 & -2.41 & -2.46 & -2.51 & -2.54 & -2.43 & -2.01 \\
\hline-1.77 & -1.12 & \multicolumn{2}{c}{-0.254} & & & & & & & &
\end{tabular}

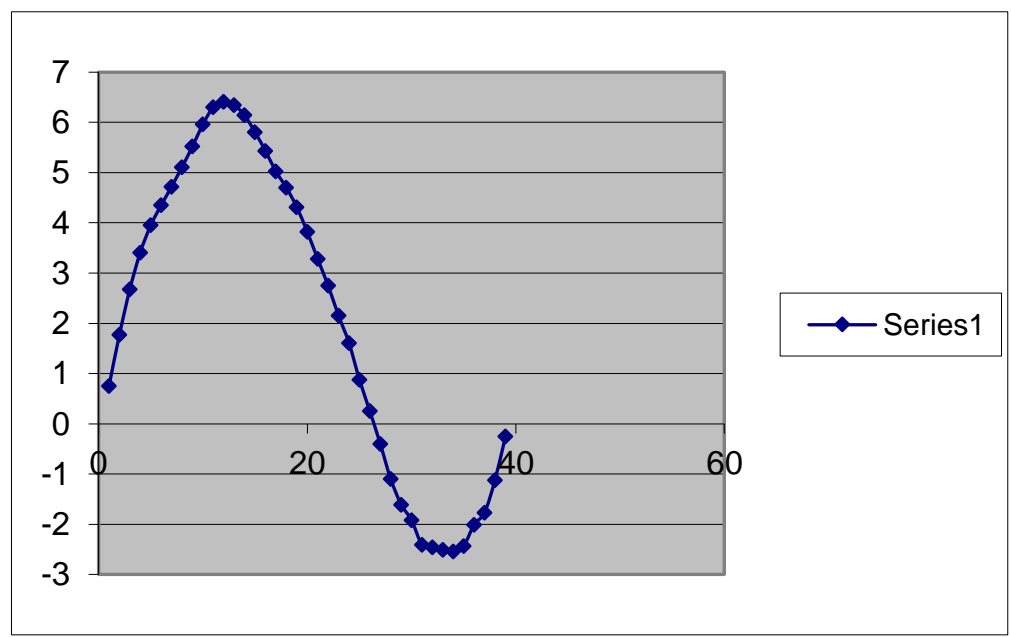

\section{CONCLUSIONS}

From the research we conclude the following

1- The sine wave function can be built using a nonlinear equation or a harmonic function with min error the efficiency of the model increases if the number of observation harmonic number and replication number Increase but this will lead to increase the computational time in the computer.

2- The reconstruction of the models that are basically build around Sine waves makes it possible to regenerate the original forms with new features added, so that it could prevent further counterfeiting.

\section{REFERENCES}

[1] H. Boche and B. Farrell, "Strong divergence of reconstruction procedures for the paley-wiener space PW $\pi$ and the hardy spaceH1," Journal of Approximation Theory, vol. 183, pp. 98-117, 2014.

[2] J. Higgins, Sampling theory in Fourier and signal analysis: foundations. Oxford University Press, 1996.

[3] K. Gr"ochenig, Foundations of Time-Frequency Analysis. Birkh“auser, 2001.

[4] I. Daubechies, Ten lectures on wavelets. SIAM, 1992, vol. 61.

[5] C. Shannon, "Communication in the presence of noise,” Proc. IRE, vol. 37, no. 1, pp. 10-21, 1949.

[6] D. Gabor, "Theory of communication. part 1: The analysis of information," Journal of the Institution of Electrical Engineers-Part III: Radio and 
Communication Engineering, vol. 93, no. 26, pp. 429-441, 1946.

[7] S. Mallat, "A theory for multiresolution signal decomposition: the wavelet representation," Pattern Analysis and Machine Intelligence, IEEE Transactions on, vol. 11, no. 7, pp. 674-693, 1989.

[8] Generation of non-linear curves using computer design phd thesis, Firas Husham Al-Mukhtar

[9] Barnett.V.D (1980) fitting straight lines the linear functional relationship with replicated observations.

[10] Sprent, P. (1981) parallelism and occurrence in linear regression.

[11]Enslein,K., Ralston , A. , \& wilf (1977) statistical methods for digital computers, New York wiley.

[12] Draper N. R , \& Smith H. (1967) Applied Reqression analysis New York wiley

[13] Otens R. k and Enochson, L.D (1972), Digital time Series Analysis, New York, John wiley \& Sons.

[14] Otnes , R.k and Enochson L.D (1978), Applied Time Series Analysis, New York, John wiley \& Sons. 